\title{
The Research on Analytical Method of Diquat Dibromide in Diquat Technical Concentrates
}

\author{
Bing $\mathrm{Gu}^{1}$, Hui Ming Xiao ${ }^{2}$ \\ ${ }^{1}$ Pesticides Test Laboratory, ShenYang Research Institute of Chemical Industry, Shenyang, 110000, China \\ ${ }^{2}$ Guidaojiaotong Polytechnic Institute, Shenyang, 110000, China
}

\begin{abstract}
The technical concentrates (TK) is prepared by technical material (TC), the content is lower than TC, it can be used for the preparation of pesticide formulations. Diquat TK is generally brown or dark brown liquid. In recent years, it develops rapidly as herbicides. This paper mainly introduces the research on analyses of the effective components in diquat TK.
\end{abstract}

\section{Introduction}

The diquat [1] is light yellow crystal, its ISO name is diquat dibromide, stable in acid and neutral solution, rapidly decomposites in alkaline conditions. Its structural formula is as follows:<smiles>CC[n+]1ccccc1-c1ccccc1Br</smiles>

\section{Experimental Part}

\subsection{Qualitative analysis}

\subsubsection{The qualitative analysis of infrared spectrum for diquat TK}

The Qualitative Analysis method [2, 3] of infrared spectrum for Diquat TK is as follows:

-Instrument: Spectrum-100 FT-IR Spectrometers.

-Scan range: $4000 \mathrm{~cm}^{-1} \sim 400 \mathrm{~cm}^{-1}$.

The diquat dibromide standard samples and diquat TK samples were prepared for the uniform transparent sheet, which will be determined of infrared spectra. The typical infrared spectrum of diquat dibromide is shown in Figure 1.

\subsubsection{The qualitative analysis for bromine ion of diquat TK}

The reagents and solutions for the qualitative analysis are as follows:

-Water: pure water;

-Potassium bromide standard;

The instruments for the qualitative analysis are as follows:

-Dionex ICS 5000 Ion chromatograph.

-Anion chromatographic column: $250 \mathrm{~mm} \times 4.0 \mathrm{~mm}$ (i.d.).

The ion chromatography operating conditions are as follows:

-Eluent: $\mathrm{KOH}$ solution

-Column temperature: room temperature

-Current velocity ( $\mathrm{mL} / \mathrm{min}): 1.2$

-Sample volume: $8 \mathrm{~L}$

-Quiescent time: $9.5 \mathrm{~min}$

The determinations of specific steps are as follows:

(1)Preparation of potassium bromide standard solution.

Take an appropriate amount of potassium bromide sample into the volumetric flask, add water to scale, and shake.

(2)Preparation of sample solution

Take an appropriate amount of diquat TK sample into the volumetric flask, add water to scale, and shake.

Under the above mentioned conditions, the retention time of bromine ion in the sample solution of potassium bromide was determined. The typical ion chromatogram for the diquat bromide ion of diquat TK is shown in Figure 2. 


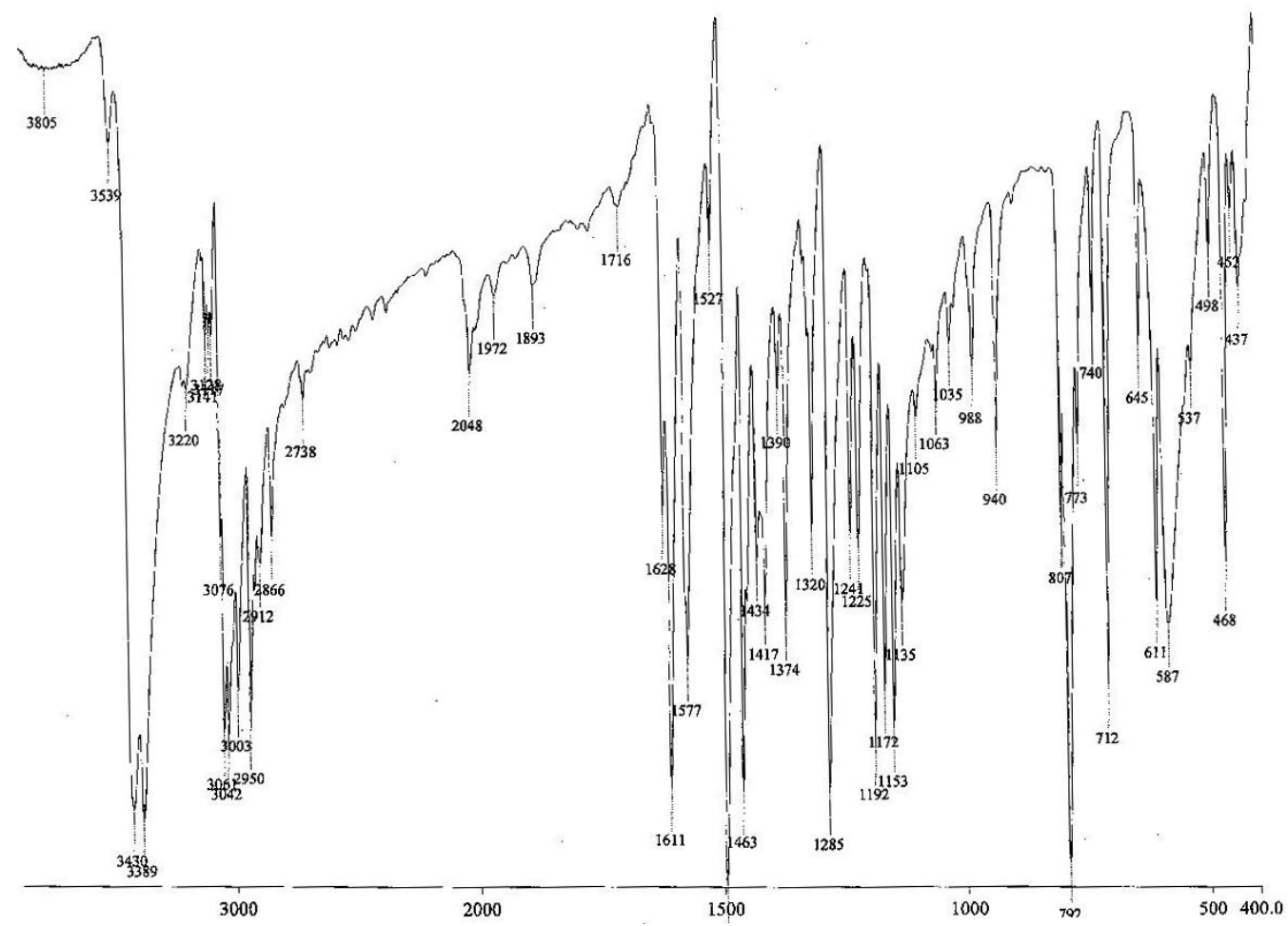

Figure 1. The Infrared Spectrum of Diquat Standard Samples.

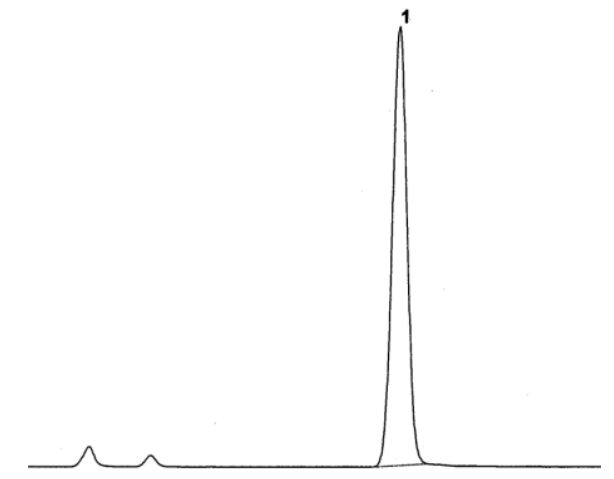

Figure 2. The Ion Chromatogram of Bromine Ion in the Sample.

\subsubsection{Liquid chromatography-mass spectrometry}

The instrument for Liquid chromatography-mass is as follows $[4,5]$ :

-Instrument: UPLC Quattro-micro Liquid mass spectrometer.

-Chromatographic column: $150 \mathrm{~mm} \times 4.6 \mathrm{~mm}$ (i.d.) stainless steel column, filled ZORBAX SB-C18, $5 \mu \mathrm{m}$ filler.

The liquid mass coupling conditions are as follows:

-Mobile phase: $\psi$ (Acetonitrile: $0.1 \%$ glacial acetic acid solution) $=5: 95$.

-Current velocity: $1.0 \mathrm{~mL} / \mathrm{min}$.

-Ion source: $\operatorname{ESI}(+)$.

-Detection range: $\mathrm{m} / \mathrm{z}$ 50 1000.

The determinations of specific steps are as follows:

(1) Preparation of standard sample solution

Take an appropriate amount of diquat dibromide samples, which are placed in the volumetric flask, adding acid aqueous solution to the scale, shake.

(2)Preparation of sample solution
Respectively take an appropriate amount of diquat TK samples, which are placed in the volumetric flask, adding acid aqueous solution to the scale, shake.

Mass spectrum determination of diquat dibromide hydrate samples, and the mass spectrum of diquat TK were shown in Figure 3, 4.

From the mass spectrum of diquat TK shown in Figure 3 and Figure 4, we can find that classic date is the same. Some special $\mathrm{m} / \mathrm{z}$ are $84.7,157.3,183.2$.

\subsubsection{Ultraviolet spectrometry}

The instrument for ultraviolet spectrometry [6] is shimadzu UV 2201 ultraviolet spectrophotometer.

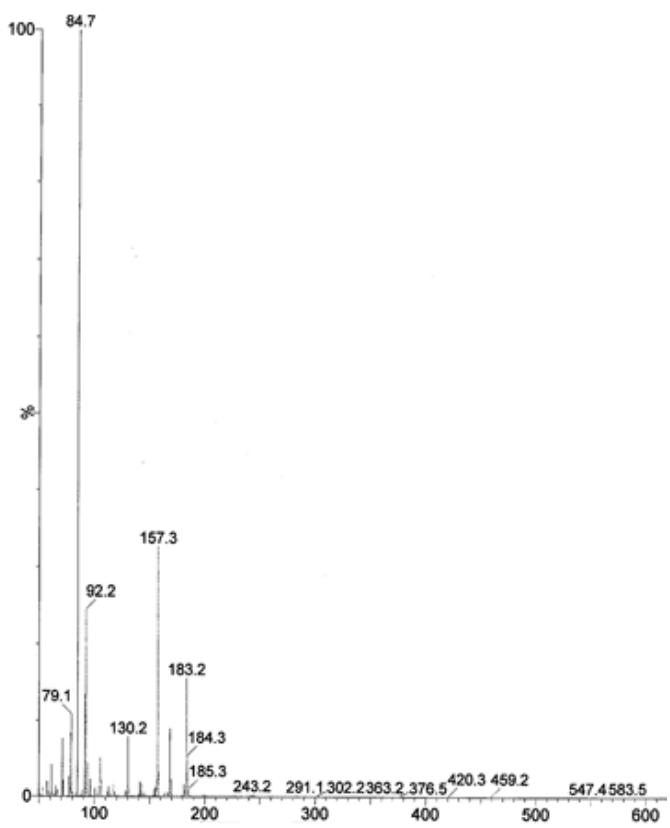

Figure 3. The Mass Spectra of Diquat Samples. 


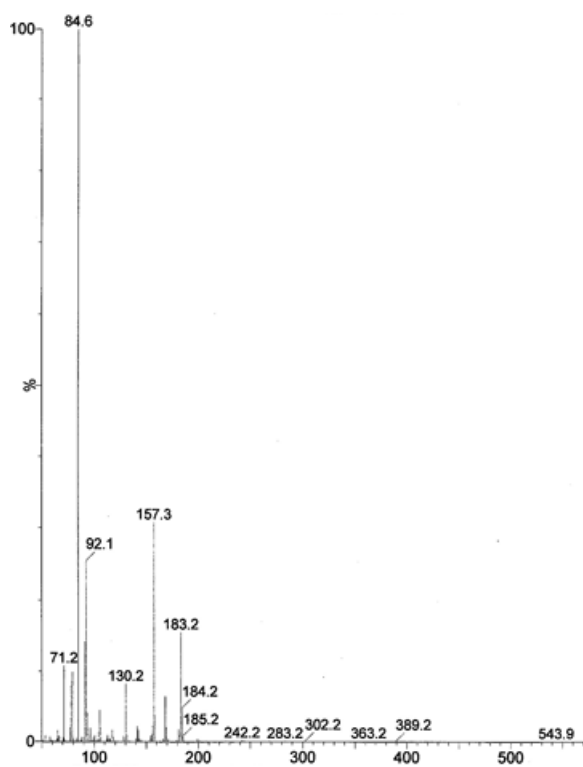

Figure 4. The Mass Spectra of Diquat in Diquat TK.

The ultraviolet spectrum qualitative conditions of diquat standard sample are as follows:

-Light source: deuterium lamp and tungsten lamp.

-Colorimetric pool: $1 \mathrm{~cm}$ quartz colorimetric cell.

-Slit: $2.0 \mathrm{~nm}$.

-Scanning range: $400 \mathrm{~nm} \sim 190 \mathrm{~nm}$.

-Scanning speed: medium.

-Scanning mode: absorbance.

-Solvent: water.

-Reference: water.

Preparation of standard sample solution: take an appropriate amount of diquat standard sample in the sample flask, dissolved with water solution and diluted to the scale, shake, and wait for detection. The typical UV spectra of the diquat standard samples are shown in Figure 5.

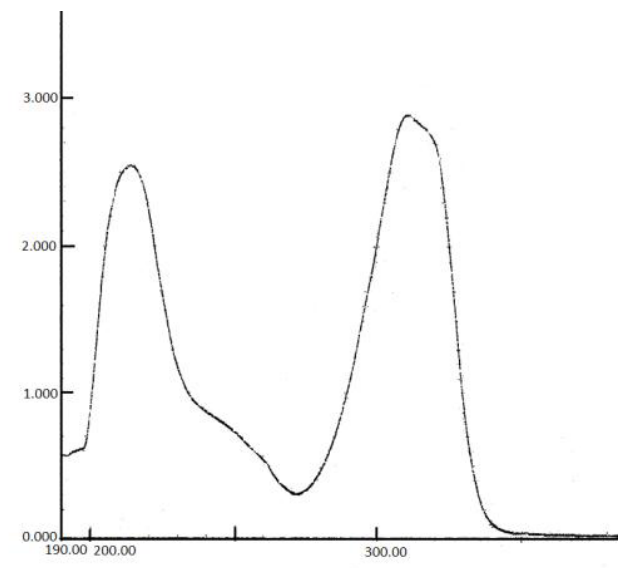

Figure 5. The Ultraviolet Spectra of Diquat Dibromide.

\subsection{Quantitative analysis}

\subsubsection{Reagents and solutions}

The specific reagents and solutions [7] are included the following contents:

-Acetonitrile: chromatographic class.
-Phosphoric acid: analytical class.

-Water: ultra pure water.

-Heptanesulfonate: chromatographic pure.

-Triethylamine.

-Phosphoric acid solution: Adjusted with phosphoric acid to $\mathrm{pH}=2.1$.

-The diquat standard samples.

Heptanesulfonate buffer solution: take an appropriate amount of heptanesulfonate, soluble in $920 \mathrm{~mL}$ ultra pure water, adding a certain amount of phosphoric acid, and then mix with triethylamine to a certain $\mathrm{pH}$ value, shake.

\subsubsection{Method summary}

Dissolving the samples in the water, make heptanesulfonate buffer aqueous solution and acetonitrile as mobile phase, stainless steel column and ultraviolet detector with $\mathrm{C} 18$ as filler (the maximum absorption wavelength is $310 \mathrm{~nm}$, as shown in Figure 5).

\subsubsection{Instrument}

Specific instruments include the following contents:

-HPLC instrument with ultraviolet variable wavelength detector.

-Column: $150 \mathrm{~mm} * 4.6 \mathrm{~mm}$ (i.d.) stainless steel column, filled with $\mathrm{C} 18,5 \mathrm{~m}$ filler.

-Quantitative sampling tube: $5 \mu \mathrm{L}$.

\subsubsection{Operating conditions}

The HPLC operating conditions [8] include the following contents:

-Mobile phase: heptanesulfonate aqueous solution acetonitrile $=92: 8$.

-Flow rate: $1.0 \mathrm{~mL} / \mathrm{min}$.

-Column temperature: room temperature.

-Wavelength: $310 \mathrm{~nm}$.

-Injection volume: $5 \mu \mathrm{L}$.

-Retention time: the diquat is about $7.8 \mathrm{~min}$.

The high performance liquid chromatography figure of typical diquat TK is shown in Figure 6.

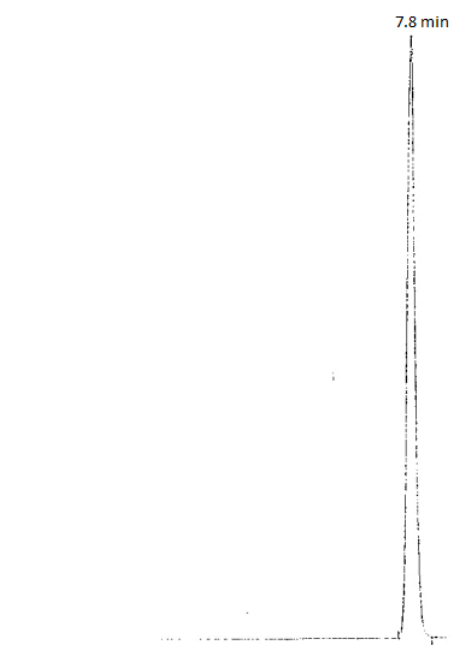

Figure 6. The High Performance Liquid Chromatography Figure of Typical Diquat TK. 


\subsubsection{Determination}

The determinations of specific steps are as follows:

(1) Preparation of standard sample solution

Take an appropriate amount of diquat standard samples into the volumetric flask, add water and shake to be dissolved, dilute with water to the scale, and shake.

(2)Preparation of sample solution

Respectively take an appropriate amount of diquat TK samples into the volumetric flask, add water and shake to be dissolved, dilute with water to the scale, and shake.

\subsection{Experimental results and discussion}

\subsubsection{Linear relationship test}

With a certain mass range, the several diquat standard samples were weighed into the different volumetric flasks which have the same volume, dissolved in water, constant volume and shake. According to the operation conditions of the method, the peak area of diquat was determined, and the average results of the two measurements were determined. The standard curve was drew, with the peak area as the horizontal coordinate, the peak area as the vertical coordinateand, the result is shown in Figure 7.

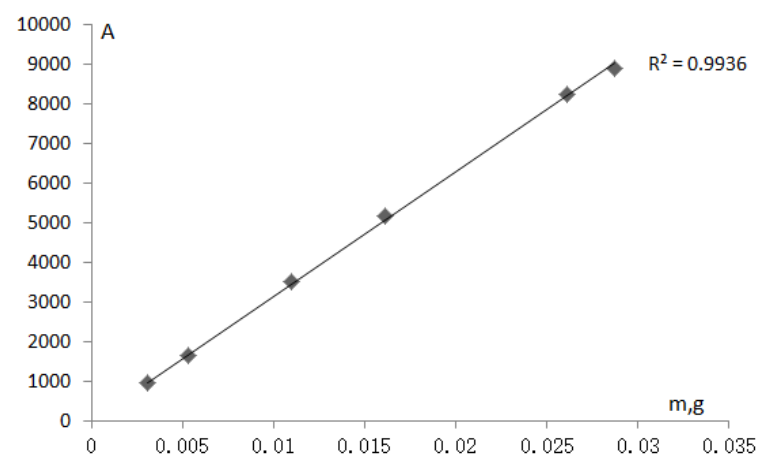

Figure 7. The Relationship Between the Peak Area and Mass Concentration of the Diquat.

From Figure 7, we can see that the diquat dibromide concentration appear a good linear relationship with the corresponding peak area in a certain range, $\mathrm{R} 2=0.9936$.

\subsubsection{Precision test}

Select a representative of diquat TK samples (marked $40 \%$ ), starting from the sample, according to the operation conditions, determination of 6 times, the results are shown in Table 1.

Table 1. The Precision Test results of Diguat TK.

\begin{tabular}{|c|c|c|c|}
\hline $\begin{array}{c}\text { Measure } \\
\text { times }\end{array}$ & $\begin{array}{c}\text { Mass } \\
\text { fraction of } \\
\text { diguat, \% }\end{array}$ & Range, \% & $\begin{array}{c}\text { average } \\
\text { value, \% }\end{array}$ \\
\hline 1 & 40.46 & \multirow{3}{*}{0.26} & \multirow{2}{*}{40.5} \\
\hline 2 & 40.58 & \\
\hline 3 & 40.71 & \\
\cline { 1 - 2 } 4 & 40.55 & & \\
\hline 5 & 40.45 & & \\
\hline 6 & 40.51 & & \\
\hline
\end{tabular}

From Table 1, it shows the method has good precision, the determination range is $0.26 \%$ with the mass fraction of diquat in diquat TK.

\subsubsection{Accuracy test}

Take a number of diquat TK together with a certain amount of diquat standard sample. According to the method for the determination of the diquat total quality and the recovery rate was calculated, and the results are shown in Table 2.

Table 2. The Diguat TK Recovery Test Results(\%).

\begin{tabular}{|c|c|c|c|c|c|c|}
\hline 1\# & 2\# & 3\# & 4\# & 5\# & 6\# & Average \\
\hline 99.93 & 100.31 & 101.02 & 99.91 & 99.82 & 99.73 & 100.1 \\
\hline
\end{tabular}

\section{Conclusion}

Firstly, using the infrared chromatography, Liquid Chromatography-Mass Spectrometry, ion chromatography, the determination of bromide ions qualitative methods analysis the diquat TK sample, and then determined by chromatographic methods diquat TK for quantitative analysis.

\section{References}

1. Kolberg, D.I.S., MacK, D., Anastassiades, M.; Hetmanski M.T., Fussell R.J., Meijer T., Mol H.G.J., Development and independent laboratory validation of a simple method for the determination of paraquat and diquat in potato [J]. cereals and pulses Analytical and bioanalytical chemistry., 404, 8 (2012).

2. Hao C., Zhao X., Morse D., Yang P., Taguchi V., Morra, F. Optimized liquid chromatography tandem mass spectrometry approach for the determination of diquat and paraquat herbicides[J]. Journal of chromatography, A: Including electrophoresis and other separation methods. 1304 (2013).

3. Abu Shawish H.M., Ghalwa N.A., Hamada M.,Basheer A. Modified carbon paste electrode for potentiometric determination of diquat dibromide pesticide in water and urine samples[J]. Materials science \& engineering, C. Biomimetic and supramolecular systems. 32, 2 (2012).

4. Xiangbing Mao, Mei Lv, Bing Yu, Jun He, Ping Zheng, Jie Yu, Quyuan Wang, Daiwen Chen. The effect of dietary tryptophan levels on oxidative stress of liver induced by diquat in weaned piglets [J]. Journal of Animal Science and Biotechnology. 01 (2015).

5. Norio TESHIMA, Tadao SAKAI. Analysis of Decomposed Species of Diquat using Photocatalytic TiO2 and its Degradation Pathway [C]. Department of Applied Chemistry Aichi Institute of Technology, 09(2006).

6. Peng Xu, Hongmei Li, Shouwei Sun, Xiefei Shi. Separation of Diquat and Paraquat by Chaotropic 
Effect HPLC [C]. Department of Applied Chemistry Aichi Institute of Technology, 09 (2006).

7. Zhang Yan, He Yong,Li Guoao, Chen Xue, Yan Lei. Application of Online Solid-phase Extraction Technology in the Field of Environmental Analysis [J]. Meteorological and Environmental Research. 05 (2014).
8. ZHAO Xiu-feng, CHEN Lin, Muhammad I A Rehmani,WANG Qiang-sheng, WANG Shaohua,HOU Peng-fu, LI Gang-hua, DING Yan-feng. Effect of Nitric Oxide on Alleviating Cadmium Toxicity in Rice [J]. Journal of Integrative Agriculture. 09 (2013). 